\title{
Brief Discussions on Vocalization of Vocal Music
}

\author{
Jin Zhou \\ Hubei Engineering University, Xiaogan Hubei, 432000, China
}

Keywords: Vocalization, Selection and use of teaching materials, Vocal music teaching.

\begin{abstract}
Voice training is the only way of singers to improve vocalization skills. This paper discusses the importance of vocalization in vocal music learning in terms of concept, classification and teaching materials of vocalization, training of vocalization skills in vocal music learning and understandings of vocal music in teaching. Vocalization is a main means and learning method for scientific and systematic mastery of singing techniques and methods by vocal music learners as well as an important learning link for the improvement of sound and voice training.
\end{abstract}

\section{Introduction}

Vocalization is a special vocal music, which is essential for vocal music learning. As a saying goes, "one cannot play a stringed instrument without the ability to tuning." It is same for vocal music. As an artistic form with cooperative operation of human organs, vocal music cannot be learned and done best in a short time. A set of complete and reasonable theories are required for guiding our learning. Vocalization is an important vocalization practice of singing with vocals. Such practice of vocalization with vocals involves both rich artistic performance and professional singing skills for music works.

\section{Concept of vocalization}

Vocalization is a form of vocal works of singing with vocals rather than specific lyrics. There are no lyrics. Vowels and vocals (a, e, i, o, u) or syllables (do, re, mi, fa, sol, la, si) are generally used for singing. Vocalization has rich artistic performance in vocal works and meanwhile contains professional singing skills. It has double professional attributes, i.e. "singing art" and "singing technique”. Such music form originally occurred not specially for music learners. It was a product of the development of vocal music art to a certain stage. To make singing voice more beautiful, artists conducted a series of studies on the human voice and singing training naturally, thus directly causing the great emergence of vocal music etudes (the predecessor of vocalization).Vocal music art has been praised highly thereafter. Vocalization has become the only way of current music lovers to learn.

Source of vocalization: (1) Artists in the early stage used "sight-singing etudes" with piano accompaniment for vocalization of vocal music.(2) Vocalization was derived from Italian (Vocalizzo) and emerged in the mid 18th century. There are no lyrics in the music. Only vowels and syllable names are used for singing. French composers J.Lully and J.Rameau created vocal works without lyrics at the earliest. More vocalization works had been created successively by the 19th century.

\section{Classification of vocalization}

\subsection{Vocalization with sound production}

Vocalization with sound production refers to a series of preparatory practice of singers before singing. Vocalization of vocal music is short, which is circulating practice of a specific sound type with vocals. It mainly aims at regulating vocal organs of human body so as to reach a natural vocal state. Meanwhile, it plays a helping role. With long-term practice, it can help students establish a good, scientific and complete vocal system, master good vocal methods and lay a solid foundation for future artistic singing. 


\subsection{Sight-singing vocalization}

Sight-singing vocalization uses some "sight-singing etudes" with piano accompaniment for voice training. Such sight-singing etudes use "syllable names" rather than "vocals" for practice. Then, melodies with only tune and without lyrics and the form of sight-singing etudes with piano accompaniment emerged, which gradually developed into a kind of vocalization.

\section{Teaching materials selected and used for vocalization}

\subsection{Foreign vocalization}

The most common vocalizations include Abt's Vocal Etude, Concone's 50 Vocal Etudes, Siber's 36 Preliminary Vocalizations, Vaccaj's Practical Vocal Etudes, Panofka's 24 Vocal Etudes and Collection of Spicker's Senior Vocal Etudes etc. These vocalizations are important systematic and standard teaching materials in professional vocal music teaching.

Abt's Vocal Etude is the most classic teaching material recognized internationally. It is divided into four parts: part I - practice of sound production and interval; part II - practice of training fluency technique; part III - practice of 20 fixed syllable names; and part IV - 12 most difficult vocalizations. The teaching material starts with the most basic vocalization practice and proceeds from the easier to the more advanced. The training of various skills is arranged through reasonable teaching so as to establish a correct and good vocal system.

Concone's 50 Vocal Etudes involve a simple style, broad melodies, steady voice and double roles in helping students make pauses and breathe correctly and thus develop a good singing style.1. This book guarantees scientific, systematic and standardized vocal music teaching: it has medium range and requires students to sing in order and step by step and not to be anxious for success in terms of its schedule arrangement.2. It plays a great role in helping singers develop a good singing style and habits: it has established a very scientific teaching system in terms of the style and melody of works, the division of phrases and the application of breath.3. It can raise the interest of singers in songs: it has very beautiful melodies and variable rhythms making classroom teaching and training no longer dry and dull. Singing of beautiful melodies not only allows singers to master skills in pleasant practice, but also builds a bridge for direct transition from voice training to song training. Both students and teachers can benefit.

\subsection{Domestic vocalizations for teaching}

Common Chinese vocal etudes include Shisanzhe Vocal Etude compiled by Wang Pinsu, 30 Articulation Vocalizations by Song Chengxian, 69 Vocalizations with Combination of Tone and Word by Shi Weizheng and Beautiful Rhyme - Vocal Music Voice Practice 123 by Wu Ying (the whole book is divided into the practice of breath, coherence, maintenance, bounce, flexibility, from high to low and rolling).These books are precious vocal music teaching data in China.

\subsection{Value and effect of vocalization}

Most vocal etudes contacted by us daily have strong pertinence. Vocal etudes undoubtedly aim at improving students' singing ability and training them so as to grasp a good singing state.Therefore, we should attach more importance to the role of vocalization in vocal music teaching, teach students in accordance with their aptitude and provide different vocal music practice for different vocal music learners. Reasonable adoption will promote the development of vocal music teaching more favorably.

\section{Training contents of vocalization}

\subsection{Pay attention to liaison training}

Liaison makes each tone without stopping. Cavities have resonance and perform their own responsibilities without change and interruption and with breath maintenance. The use of liaison in a phrase aims at making the voice mellow and full and keeping a smooth voice line. There are stricter 
requirements for liaison in vocal works. Liaison should be smoothing. Such connected phrases require breath control and the unification of cavities with resonance.

Practice of vocalization:

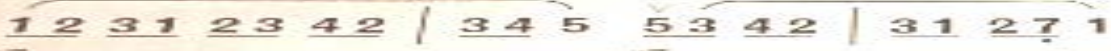

1) In the first practice, attention should be paid to the coherence of voice. It is required to take a breath where breathing symbol ${ }^{\sim}$ is given. The voice should be smooth and steady at a low speed. Requirements for other vowels are same.

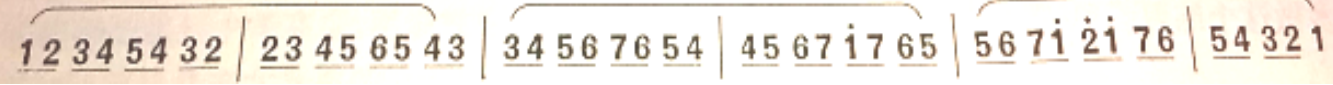

2) In the second practice, attention should be paid that mouth, lip and tongue shall be natural and steady. During practice, words should be read out gently, slowly and clearly with laryngeal cavity fully relaxed.

\subsection{Pay attention to leap training}

Leap is a bounce method making voice more flexible. It is of great help to the improvement of students' breath, the control of dynamics and their experience and seeking for high position. When singing leap, students should mainly pay attention to make each tone leap clearly and smoothly with breath. Leap requires granularity of voice which should be made rapidly and accurately like table tennis.

\begin{tabular}{llll|llll|l}
\hline & 3 & 5 & 5 & $i$ & 5 & 5 & 3 & 1
\end{tabular}

1) In the first leap practice, the voice should be short, rapid, flexible and smooth during singing.

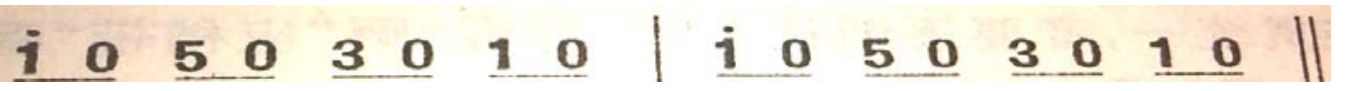

2) The second etude involves the practice of a large vocal range and requires deep breath and clear articulation. The voice should be short, rapid, flexible and smooth.

\subsection{Pay attention to the training of open cavities}

Cavity is a priority among priorities in vocal music learning. Cavities generally include thoracic cavity, head cavity, pharyngeal cavity and oral cavity etc. These cavities are main channels for singing resonance. During voice training, it extends to each resonance cavity above thoracic cavity with the rise of pitch, enters into pharyngonasal cavity and head cavity naturally along pharyngeal cavity channel and then gets to head cavity for resonance. The key to obtain the resonant position of head cavity is to "open head cavity and centralize tone”. Singing is a general movement. It is a project with simultaneous cooperative operation of a series of sound production parts such as articulation, sound production, resonance and breath.

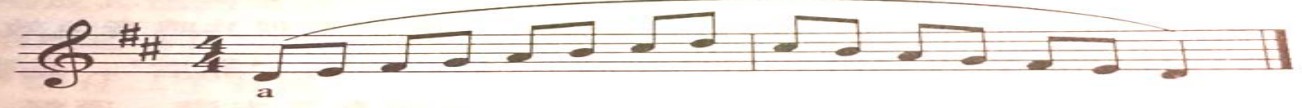

In the practice of this vocalization, vowels a and u should be used. It is required to open oral cavity, breathe deeply and make the voice smooth.

\subsection{Pay attention to the training of voice training position}

With respect to the regulation of sound position, many vocal music teachers use the method of humming to find the position rapidly. Humming practice is good for obtaining a high and front sound position, which can make laryngeal cavity and oral cavity produce sound naturally. Method and key points for high position of sound production: 1. vocalization practice with alternative humming with open mouth and closed mouth can keep the concentration point of voice in the highest part of head 
cavity.2. When the tone rises gradually, the concentration point of voice moves upward along hard palate gradually.3. "Singing above breath foil” refers to singing discharged along breath and guiding the voice to be centralized and in the front.

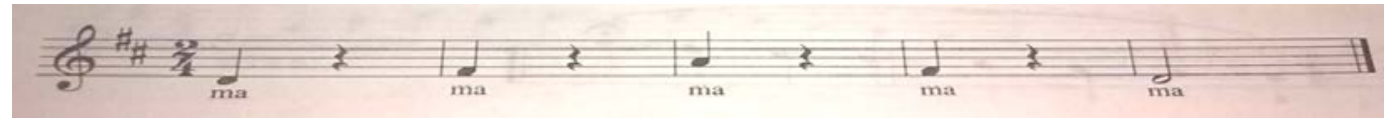

1) In the first practice: 1 . Inhale calmly at lower abdomen; 2. Keep mandibular joint, jaw and front neck relaxed; 3. Vocalize at the second fastener on the pit of the stomach; 4. Vocalize at the pit of the stomach as "vertical sigh" when starting voice.

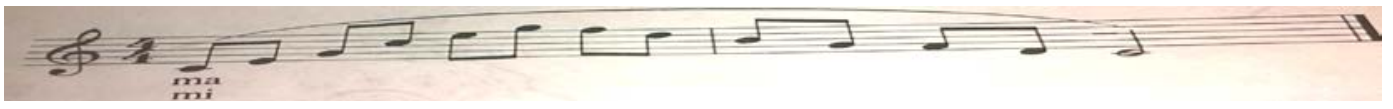

2) The second practice requires opening the throat just as “inhaling” and singing close to pharynx wall, thus making the whole pharyngeal cavity smooth.

\subsection{Pay attention to breath training}

The position of voice is abstract, but position is a specific material phenomenon. For example, action of soft palate, oral cavity opening and external action of breath etc. are decisive factors for accurate position of voice. The correctness of breathing method in position training is the first condition for reaching the accurate position of voice. The voice will not be beautiful without adequate support of breath. Even if it enters the right position, once breath is insufficient to support the continuity of voice, its position will change and voice will fall off.

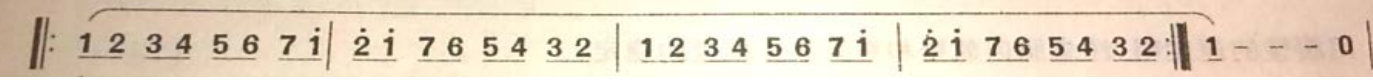

This etude requires singing in a low voice, paying attention to control volume, regulating breath, keep pitch position and singing under the condition of smooth breath.

\section{Vocal etudes in teaching}

\subsection{Disadvantage of vocalization: it pays too much attention to sound production itself and neglects music.}

In vocal music learning, some vocal music teachers always pursue for voice quality of students in the process of vocalization, but neglect the training of comprehensive ability of music. Therefore, students lack the performance of music in actual singing and it is difficult for them to realize resonance of cavities and fully express connotations and musical emotion of works.

In the teaching process, it is required to make students pay attention to all terms on works such as dynamics, speed and strength during vocalization. Meanwhile, teachers should explain some background of work production to students so that they can have unique perception and expressive force of music.

\subsection{Application of vocalization in vocal music teaching}

\subsubsection{Avoid selecting vocalization blindly}

Vocalization refers to teaching etudes with "teaching effect". The responsibility of teachers is to guide students for vocal practice with correct methods. Teachers should understand students' tone quality, characteristics and basic voice state, select etudes appropriate to students' conditions and require students to start the practice of basic vocalization skills with natural vocal zone, transit to middle pitch part and gradually extend vocal range after the voice in middle pitch part is steady and smooth. Students should select vocal works appropriate to themselves on purpose so as to improve their own abilities and level.

\subsubsection{Pay attention to the length of vocalization time}


In our daily vocal music teaching, some teachers spend a lot of time on the link of vocalization. After long-time vocalization, the voice of students at a slightly lower level has been extremely tired. This cannot make students progress better. Therefore, teachers are expected to arrange vocalization and singing reasonably, exploit students' individual features for training and education and teach them in accordance with their aptitude in the teaching process 0 \} so that students can learn better.

\subsection{Pay attention to keep the unification of cavity and position in the vocalization process}

Cavity and position are specialized terms in vocal music. To be frank, cavity is "the voice box producing resonance" and position is "the place of tone". To have a good resonance effect, singing should have three important factors, i.e. appropriate support by deep breath, correct vocalization position and accurate resonant cavity. Their cooperation requires uniform arrangement and management in psychology so as to unify resonant cavity and position and obtain a uniform tone.

\subsection{Strengthen accompaniment of etudes appropriately}

It is very necessary to strengthen accompaniment. Singers and piano accompaniment play an equally important role in vocal music singing. How can a singer have good performance if piano accompaniment is always wrong? In a word, sentimental and passionate accompaniment is of great help to singers and can stimulate the emotion of singers and present the song and emotion better.

\section{Conclusion}

Vocal music is an abstract science of art with great difficulty and high practicalness. As an old saying goes, "the edge of a treasured sword sharpens by grinding." Vocal music, a discipline highly combining art and technique, requires us to accumulate bits and pieces in learning and practice and experience each link with all heart. Reasonable use of vocalization has self-evident significance for vocal music teaching. Music learners should listen, ask, think and summarize more in the learning process of vocal music and march towards new objectives step by step.

\section{References}

[1] Shen Xiang. Shen Xiang's Vocal Music Teaching Method.Shanghai. Shanghai Music Publishing House. 1998.

[2] Shi Weizheng. Basis of Vocal Music. Beijing. People’s Music Publishing House. 2002.07.

[3] Zou Benchu. Singing. Study on Shen Xiang’s Singing System. Beijing. People’s Publishing House. 2000.11.

[4] Shen Xiang. Vocal Music Teaching Art. Shanghai. Shanghai Music Publishing House. 1998.11.

[5] Yu Renhao. Introduction to Musicology. Beijing. People’s Music Publishing House. 2003.

[6] Lin Junqing. Eight Major Steps of "Pharyngeal" Practice. Shanghai. Shanghai Music Publishing House. 1999.

[7] Zhou Xiaoyan. Basis of Vocal Music. Higher Education Press. 1990.

[8] Abt’s Vocal Etude. Beijing. People’s Publishing House. 2006.05.01.

[9] Paper of Professor Wang Yueping, Importance of Vocal Music Teaching in Vocalization.

[10] Shen Xiang. Study on Singing System. Beijing. People’s Publishing House. 2000.11. 